This is the author's version of the work. It is posted here by permission of the AAAS for personal use, not for redistribution. The definitive version was published in Science , (2021-04-16), doi: 10.1126/ science.abf4588

\title{
Neuropixels 2.0: A miniaturized high-density probe for stable, long-term brain recordings
}

Nicholas A. Steinmetz ${ }^{*,+1,2}$, Cagatay Aydin*,3, Anna Lebedeva*,4, Michael Okun ${ }^{*, 5,6}$, Marius Pachitariu ${ }^{*, 7}$, Marius Bauza $^{4}$, Maxime Beau ${ }^{8}$, Jai Bhagat ${ }^{6}$, Claudia Böhm ${ }^{7}$, Martijn Broux ${ }^{3}$, Susu Chen ${ }^{7}$, Jennifer Colonell ${ }^{7}$, Richard J. Gardner ${ }^{9}$, Bill Karsh , Fabian Kloosterman ${ }^{3}$, Dimitar Kostadinov ${ }^{8}$, Carolina Mora-Lopez ${ }^{10}$, John O'Callaghan ${ }^{10}$, Junchol Park ${ }^{7}$, Jan Putzeys ${ }^{10}$, Britton Sauerbrei ${ }^{7}$, Rik J. J. van Daal ${ }^{11,3,14}$, Abraham Z. Vollan ${ }^{9}$, Shiwei Wang ${ }^{10}$, Marleen Welkenhuysen ${ }^{10}$, Zhiwen Ye ${ }^{2}$, Joshua Dudman ${ }^{7}$, Barundeb Dutta ${ }^{10}$, Adam W. Hantman ${ }^{7}$, Kenneth D. Harris $^{6}$, Albert K. Lee ${ }^{7}$, Edvard I. Moser ${ }^{9}$, John O’Keefe ${ }^{4}$, Alfonso Renart ${ }^{12}$, Karel Svoboda ${ }^{7}$, Michael Häusser ${ }^{8}$, Sebastian Haesler ${ }^{3,13}$, Matteo Carandinit ${ }^{\dagger, 1}$, Timothy D. Harris ${ }^{\dagger, 7,15}$

* Co-first authors

†Corresponding authors: nick.steinmetz@gmail.com, m.carandini@ucl.ac.uk, harrist@janelia.hhmi.org

\section{Affiliations}

${ }^{1} \mathrm{UCL}$ Institute of Ophthalmology, University College London, London, UK

${ }^{2}$ Department of Biological Structure, University of Washington, Seattle, WA, USA

${ }^{3}$ Neuroelectronics Research Flanders, Leuven, Belgium

${ }^{4}$ Sainsbury Wellcome Centre, University College London, London, UK

${ }^{5}$ Centre for Systems Neuroscience and Department of Neuroscience, Psychology and Behaviour, University of Leicester, Leicester, UK

${ }^{6} \mathrm{UCL}$ Queen Square Institute of Neurology, University College London, London, UK

${ }^{7}$ Janelia Research Campus, Howard Hughes Medical Institute, Ashburn, VA, USA

${ }^{8}$ Wolfson Institute for Biomedical Research, University College London, London, UK

${ }^{9}$ Kavli Institute for Systems Neuroscience, Norwegian University of Science and Technology, Trondheim, Norway

${ }^{10}$ IMEC, Kapeldreef 75, 3001 Leuven, Belgium

${ }^{11}$ ATLAS Neuroengineering, Leuven, Belgium

${ }^{12}$ Champalimaud Centre for the Unknown, 1400-038, Lisbon, Portugal

${ }^{13} \mathrm{VIB}$, Leuven, Belgium

${ }^{14}$ Micro- and Nanosystems, KU Leuven, Leuven, Belgium

${ }^{15}$ Department of Biomedical Engineering, Johns Hopkins University, Baltimore, Maryland 


\section{Abstract}

Measuring the dynamics of neural processing across timescales requires following the spiking of thousands of individual neurons over milliseconds and months. To address this need, we introduce the Neuropixels 2.0 probe together with novel analysis algorithms. The probe has over 5,000 sites and is miniaturized to facilitate chronic implants in small mammals and for recording during unrestrained behavior. High quality recordings over long timescales were reliably obtained in mice and rats in six laboratories. Improved site density and arrangement combined with new data processing methods enable automatic post-hoc correction for brain movements, allowing recording from the same neurons for over two months. These probes and algorithms enable stable recordings from thousands of sites during free behavior even in small animals such as mice. (125/125)

\section{Introduction}

A major challenge for neuroscience is developing tools to record neuronal activity at large scale and across all relevant timescales (1-5). Recent advances, including the Neuropixels probe, have leveraged CMOS fabrication methods to significantly expand the number and density of recording sites $(6,7)$, allowing unprecedented recordings of large populations of neurons distributed across the brain at single spike resolution (8-11). The Neuropixels probe has seen rapid adoption and wide application in diverse species including mice (12-21), rats (22-25), ferrets (26), and non-human primates (27). Nevertheless, key barriers still prevent the recording of individual neurons stably over long timescales of weeks to months, of large neuronal populations in small animals that are freely behaving, and of neurons packed densely in brain structures with diverse geometries.

The ultimate aim of chronic recordings is to record from the same neurons over days and weeks, but this goal has been difficult to achieve for large populations of neurons. Recording individual neurons stably over weeks or months is critical to understand processes that evolve over time, such as learning, memory, and plasticity. To provide such stable recordings and minimize tissue damage, considerable effort has been devoted to developing probes that are flexible (28-30) and/or <10 $\mu \mathrm{m}$ in size (31-34), but these approaches make insertion difficult and limit the number of recording sites per inserted shank. Moreover, high quality signals can be recorded for more than eight weeks even with relatively rigid and large devices such as wire tetrodes $(35,36)$, Utah arrays $(37,38)$, traditional silicon probes (39-41), and Neuropixels probes $(6,25,42)$. However, neither flexible nor rigid devices have been able to consistently record large numbers of identified individual neurons over weeks or months (28, $36,39,43-48)$.

Rodents, especially mice, have become the dominant mammalian species for studying the neural basis of behavior, but their small size has made it challenging to record large populations of neurons during unrestricted movement. Implants that can be carried without impeding the behavior of a mouse must weigh less than $\sim 3 \mathrm{~g}$ and span less than $\sim 2 \mathrm{~cm}$ in height, and must connect with thin, flexible cables (or wirelessly). These limitations have precluded the use of many high-count electrode arrays (49-51). Even the relatively small Neuropixels probes, which permit chronic recording in freely-moving mice, can cause some impediments to movement (42), indicating the need for still smaller devices. 
Finally, while single-shank silicon probes like Neuropixels achieve dense coverage along a line, some brain structures are more effectively recorded with other geometries. Several techniques including the Utah array, tetrode arrays, and microwire arrays can sample across a plane approximately parallel to the brain surface ( 35 , 37, 52-54). However, to record in layered or deep structures such as isocortex, striatum, hippocampus, or superior colliculus, it can be ideal to densely sample a plane perpendicular to the brain surface (49-51).

To address these challenges, we developed the Neuropixels (NP) 2.0 probe and open-source software for motion correction and spike sorting. To record individual neurons stably across months, the probe has a denser, linearized geometry which allows the software to perform post-hoc motion correction. To record in small, freelymoving animals, the probe and headstage were miniaturized to $\sim 1 / 3$ size, so that two probes and their headstage weigh 1.1 g with no loss of channel count (384 channels / probe, where 'channel' refers to a signal processing and data transmission path). A 4-shank version of the probe can densely sample activity from a $\sim 1 \times 10 \mathrm{~mm}$ plane with 5,120 recording sites (where 'site' refers to a physical electrode location along a probe shank). Two of these probes thus have 10,240 sites, 768 of which are recordable simultaneously through a single headstage. New implantation hardware allows recovery and re-use of these implanted probes. Finally, we demonstrate a recording scheme in which multiple sites are recorded concurrently on a single channel, allowing the probe to record neurons with large spikes from a wider span of brain tissue. These advances enable stable, long-term measurements of neuronal activity at an unprecedented scale.

\section{Results}

The NP 2.0 device design is miniaturized and optimized for long-term stable recordings in small mammals (Fig 1a). Like NP 1.0 probes, the NP 2.0 probe has a rigid base and $4 \mathrm{~cm}$-long flexible cable that attaches to a headstage. The rigid area is shorter and narrower near the tip to facilitate close positioning of multiple probes. The headstage is also miniaturized, and serves two probes at once. Together, two probes plus a headstage weigh $\sim 1.1 \mathrm{~g}$, suitable for chronic implantation and freely-moving recordings in a mouse. The recording sites are denser with center-to-center spacing of $15 \mu \mathrm{m}$ along the vertical dimension, compared to $20 \mu \mathrm{m}$ for NP 1.0. Thus the number of sites per shank is 1,280 rather than 960, and there are both single- and 4-shank versions, with 5,120 recording sites in the latter. The recording sites are vertically aligned in two columns rather than staggered along the shank (Fig 1a), which is critical for the motion correction algorithm described below.

Despite being miniaturized, NP 2.0 probes each have 384 simultaneously recordable channels, with improved ADC resolution. The probes output a single wide-band 14-bit data stream (Fig 1b). The light sensitivity of NP 2.0 is as low as NP 1.0 (Fig S1), and noise levels are slightly increased (from 5.4 to $7.2 \mu \mathrm{V}$ root mean square voltage for the 'alpha' probes reported here, measured for the recording channel without electrode site noise). Similar to recordings with NP 1.0, well-isolated individual neurons can be distinguished on overlapping channels (Fig 1cd).

Programmable switches allow rapid remapping of the recording channels to the recording sites, enabling recordings from thousands of sites per experiment (Fig 1e,f). The switches can be reset from the recording software in $<1 \mathrm{~s}$. To illustrate this, we recorded from 6,144 sites, 768 at a time, from a pair of probes with a 
single headstage in a freely-moving mouse (Fig 1e). By configuring the switches to record from a selection of sites across shanks, the 384 recorded channels of a single 4-shank probe can sample from sites densely covering a plane spanning $750 \times 720 \mu \mathrm{m}$, an arrangement especially suited to structures oriented perpendicular to the brain surface (Fig 1f). This mode enables reliable observation of activity dynamics such as sequences in dense local populations.

NP 2.0 probes provided reliable recordings from large populations of neurons with recoverable probes in both rats and mice, with little indication of an upper limit to the duration of these recordings. We confirmed the quality of their recording characteristics over at least 8 weeks, as done for NP 1.0 probes $(6,25,42)$, by implanting them chronically in 21 rats and mice in 6 labs. Twenty of the 21 implants were successful and recorded neurons until the experiment was ended at the discretion of the experimenter (Table S1). Large-amplitude spiking activity was maintained consistently over 8 weeks (Fig 2a) and consistent firing patterns were maintained across the depth of an example recording for $>44$ weeks (Fig 2b). Most chronically implanted probes across laboratories gave good recordings for at least 8 weeks as measured by the stability of total recorded firing rates (Fig 2c; for $n=18 / 24$ recordings the null hypothesis that firing rates were not declining with time could not be rejected; $p<0.05$, t-test for correlation coefficient over days, Fig 2d) and of spike-sorted neuron count (Fig 2e; for $n=19 / 24$ recordings the null hypothesis that neuron count was not declining with time could not be rejected; $p<0.05$, t-test for correlation coefficient over days, Fig 2f). Seven of the 21 implants were performed with custom 3D printed fixtures (Fig S2), which protected the probe and headstage and enabled probe recovery after the experiment. After recovery and cleaning, these probes were re-implanted in new subjects. In total, 7 out of 8 probes implanted with hardware suitable for recovery were recovered in working condition (Table S1). Finally, in one lab, recordings were made for more than 150 days in all implants $(n=3)$, with a maximum time from implant to recording of 309 days, while retaining high firing rates, high neuron counts, and high quality individual neurons even at this late time point (Fig S3).

To record individual neurons stably over short and long timescales, it is necessary to maintain detection of spikes from the neuron over time and to match the spikes to the same unit. Classically, a neuron's spikes are observed to decrease amplitude and disappear over a recording session or over longer timescales. We hypothesized that this amplitude decrease may be generally due to relative motion between the brain and the probe over time (Fig $3 a$, blue arrows). With a small number of recording sites, a neuron that moves relative to the probe will be lost, but with a large number of sites the neuron will simply be recorded on different sites provided that motion is parallel to the probe. We expect motion primarily along this axis because resistance to movement is much greater in axes perpendicular to the probe, which would require the probe to sever the surrounding tissue. Indeed we commonly observed consistent shifts in spiking patterns along the length of the probe in many of our recordings (Fig 3b).

We therefore asked whether spikes from individual neurons were preserved and detectable even when the brain moved relative to the probe, and whether we could correct for the effects of this motion. To test this, we devised an approach to give us ground truth knowledge of the motion of the probe relative to the brain. We performed acute recordings in awake, head-fixed mice while moving the probe up and down programmatically via an 
electronic micromanipulator that imposed a known pattern of motion relative to the brain (Fig 3a, red arrows; 10 cycles of triangle wave movement with amplitude $50 \mu \mathrm{m}$ and period $100 \mathrm{~s} /$ cycle).

We then applied to the ground-truth datasets an unsupervised algorithm to correct motion in recordings posthoc. The algorithm, implemented in the Kilosort 2.5 software package, determines the motion over time from the spiking data (Fig $3 \mathrm{c}, \mathrm{d})$ and corrects it with spatial resampling of the original raw data, as in image registration (Fig 3c,e; see Methods). Before applying the algorithm, datasets with imposed motion clearly reflected the triangle wave pattern (Fig $3 f$ ). The algorithm successfully estimated this imposed motion (correlation between known probe position and estimated position from the algorithm $=0.74,0.79$, and 0.59 in $n=3$ recordings; Fig $3 \mathrm{~d})$. Applying the algorithm to the raw data and then re-detecting spikes removed the relative motion between the brain and the probe, resulting in stable patterns of spiking activity over time (Fig $3 \mathrm{~g}$ ).

Confirming the quality of the correction, we found that each neuron's spikes were detected independent of probe motion by computing a correlation coefficient between each neuron's firing rate and the imposed probe position. If the spikes from a neuron are gained or lost when recording sites come closer or further from it, the observed firing rate would correlate with the time course of the imposed motion. Conversely, if the algorithm successfully corrects the motion, this correlation coefficient should be minimized. The algorithm significantly reduced this correlation, decreasing it to near chance levels (Fig 3h; absolute value of $0.224 \pm .007$ mean \pm s.e.m. before

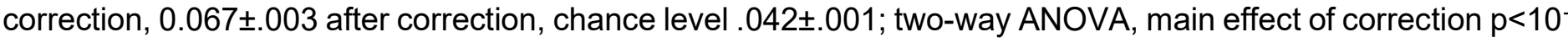
$\left.{ }^{10}\right)$. Moreover, the algorithm markedly improved yield of neurons with stable firing rates ( $n=3$ separate recordings yielded $n=156,103$, and 22 stable units without correction versus $n=181,201$, and 108 stable units respectively following correction; corresponding but opposite changes in the number of unstable units; Fig 3i).

Applying post-hoc motion correction also improved firing rate stability and neuronal yield in data obtained under the same paradigm with NP 1.0 probes, though the firing rate correlation after correction was significantly higher

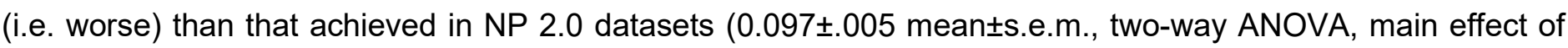
probe type $p<10^{-10}$; data not shown). Motion correction was presumably more successful in NP 2.0 datasets because of the vertically aligned sites and smaller gaps between sites (Fig 1a), which together increase the spatial resolution of sampling along the direction of motion.

We then improved this motion correction algorithm to correct not only the relative movements between the brain and probe that occur on a fast time scale but also those that occur across days. Given that NP 2.0 probes record from neurons stably even as the brain moves relative to the probe during a session, we reasoned that we could apply a similar approach to spiking activity recorded across multiple sessions over weeks or months. Indeed, as in the acute situation, the spiking activity measured across weeks appeared to represent the same patterns but shifted in depth (Fig 4a). To track neurons across sessions, we implemented a version of the motion correction algorithm that inferred and corrected the shift only at the point where datasets from the two days are spliced together, i.e. at the end of the first and start of the second dataset (see Methods). Following this step, spikes were sorted together across the splicing point, using only the shapes and spatial footprints of the waveforms. Thus, spikes were joined into single clusters across days without reference to their functional properties. We then focused the subsequent analyses on the $75 \pm 16 \%$ of the units that were deemed to be active and well 
isolated across pairs of recording sessions, and could thus potentially correspond to the same neuron across sessions.

With this improved algorithm for motion correction, we were able to record from the same neurons in visual cortex across weeks. To establish a ground-truth metric for whether a group of spikes (a "unit") recorded across different days corresponded to the same neuron, we relied on the fact that neurons in primary visual cortex have visual responses unique amongst their neighbors (55-59). Here, we used a battery of 112 natural images to establish a visual fingerprint of a large subset of units (Fig 4b,c). We then assessed whether the algorithmicallytracked units represented the same neuron by determining whether their responses to our battery of images across two sessions were more similar to each other than to the responses of the nearest other unit. This assay revealed that most units are successfully tracked across days (Fig 4d) and weeks (Fig 4e). For recording sessions that are 16 days apart or less, an estimated $93 \% \pm 9 \%$ of well-isolated units were successfully tracked in time (mean \pm s.d. across 36 shanks; $n=1,110$ units, 15 sessions in 3 mice; Fig 4f). Across sessions separated by 3-9 weeks, we could still successfully track $85 \% \pm 19 \%$ of the well-isolated units ( $n=638$ units, 30 shanks, 11 recordings in 3 subjects, Fig $4 \mathrm{f}$ ). In one of the three mice we observed a discontinuity: a loss of almost all of the tracked units, which we speculate may have been due to a non-coaxial shift of the probe relative to the brain. However, even in this mouse we were able to track units across pairs of recording days that were on the same side of the discontinuity. Across all analyzed recording pairs, the fingerprint similarity decreased with longer gaps between two recordings (Fig S3d). This result suggests that the representation of images in visual cortex, while overall stable $(39,48,60)$ may exhibit some drift $(61,62)$. Neuropixels 2.0 probes, together with our motion correction algorithm, allows studying questions of population coding over timescales of learning and plasticity.

Finally, we reasoned that the high signal-to-noise ratio (SNR) of many recorded neurons may allow a strategy for increasing recording coverage, in which the signals from multiple distant recording sites are combined on a single recording channel (Fig 5a). From physical considerations, connecting two distant sites to one recording channel ought to average the signals at the two sites, in principle allowing for recording from twice as many sites as there are channels (63). This strategy would reduce signal magnitude by a factor of 2 and change the noise level according to the equation:

$N_{C}=\sqrt{N_{A D C}^{2}+\left(\frac{N_{1}}{2}\right)^{2}+\left(\frac{N_{2}}{2}\right)^{2}}$

where $N_{c}$ is the noise level when recording on banks 1 and 2 combined, $N_{A D C}$ is channel noise from the recording system, and $N_{1}$ and $N_{2}$ represent noise from biological and physical sources at each electrode site (assumed uncorrelated). The SNR would thus decrease by a factor of $\sqrt{2}$ (if $N_{1}$ and $N_{2} \gg N_{A D C}$ ). However, although the SNR of each neuron will decrease, the number of recorded sites will double, yielding a viable strategy for scaling recording beyond limits on the number of recording channels.

This strategy however poses the challenge of unmixing signals from the pooled sites, which we addressed in hardware through the mapping of sites to channels and in software by analyzing spatial continuity. In the singleshank version of NP 2.0 probe we allowed for connecting multiple sites to a single recording channel while 
scrambling the arrangement of sites from one bank of 384 sites to the next (Fig $5 a)$. Because the spikes of a neuron are typically recorded by multiple contiguous sites, the pattern of observed waveforms across channels from one neuron will form a spatially compact group only when interpreted as arising from one bank, and not the other (Fig S6a). To classify recorded neurons to channel banks, we devised a "mismatch score" that measures the dispersion of waveforms across sites under each bank's channel mapping, with low scores indicating compact waveforms. This procedure could reliably identify which bank each neuron was recorded on: recordings made truly on just one bank resulted in nearly all neurons correctly classified as arising from the recorded bank rather than from the other (Fig S6b; 630/632, 99.7\% of neurons, $n=4$ recordings; see Methods for detailed criteria).

Using this strategy, we could reconstruct the pattern of spiking activity across 768 recording sites simultaneously with only 384 channels (Fig 5b). As expected, the spikes recorded in this double-bank configuration had half the amplitude than those in the single bank configuration. Moreover, the noise level on the combined channels closely matched the prediction of the equation given above ( $r=0.92$ and 0.95 in two recordings; Fig S6c). The SNR of spikes recorded in the double-bank configuration was $63.5 \pm 0.3 \%$ that of the single bank configuration (mean \pm s.e., $n=1,536$ recording sites, 2 recordings). As a result, the recording yielded fewer sortable single neurons than recordings performed in each bank separately (summed yields of 215, 139, 40, and 237 neurons for separate banks versus $75,44,20$, and 29 for combined banks). This strategy is therefore not optimal for recording from more neurons than on a single bank alone. Nevertheless, these neurons were recorded simultaneously across a span of the brain twice as long as that covered by a single bank of sites. This strategy enables simultaneous recording across more sites than available channels, an approach suitable for capturing neurons with large SNR over a large spatial extent.

\section{Discussion}

In summary, we have demonstrated a suite of electrophysiological tools: a miniaturized high-density probe; recoverable chronic implant fixtures; software algorithms for fully-automatic post-hoc computational motion correction; and a strategy for extending the number of recorded sites beyond the number of available channels. We presented experiments that validate the quality of acute and chronic recordings across six laboratories, and their stability over timescales of months. Finally, we provided ground-truth proof of the efficacy of motion correction on both short and long timescales. Together these tools enable an order of magnitude increase in the number of sites that can be recorded over long timescales in small animals such as mice, and the ability to record from them stably. Such recordings offer multiple advantages over those obtained chronically with 2-photon imaging: much higher temporal resolution, light weight of the implant, and ability to access neurons in multiple deep structures with minimal tissue displacement.

While our approaches for computational motion correction achieved marked improvements over previous algorithms (Figs. 3,4), we did not completely eliminate instability (Fig 3f) and we could not record every neuron stably over long timescales (Fig 4d). The remaining instability could be due to experimental factors: mechanical forces in experiments with imposed probe motion might alter neuronal firing of nearby neurons; and neuronal 
death or true changes in visual response properties $(61,62)$ could result in failure to track neurons over weeks. The remaining instability might also be due to imperfect spatial sampling. Biophysical models of neurons' extracellular potentials (64) predict features of these potentials that are smaller than the sampling density of the probe $(15 \mu \mathrm{m})$, suggesting that probes of still higher density (65) may in the future yield even better solutions to these challenges. 


\section{Materials and Methods}

\section{Neuropixels 2.0 device design}

The Neuropixels 2.0 probe consists of one or four shanks (i.e., the thin segment inserted into the brain) and a base (containing the electronics for filtering, amplification, multiplexing, digitization, and power management), fabricated with $130 \mathrm{~nm}$ CMOS process as one piece (66). The base is affixed to a rigid printed circuit board (PCB) and a thin flexible ribbon cable ("flex cable") that plugs into a headstage. From the headstage, a $5 \mathrm{~m}$ cable runs to a custom PXIe ("Peripheral Component Interconnect (PCI) eXtension for Instrumentation"; a standardized modular electronic instrumentation platform) data acquisition card (67) which connects to a computer via an offthe-shelf PXI chassis (e.g. NI 1071, National Instruments), and custom software collects the data and writes to disk. Each of these system components are described in turn below. The details presented here apply to the "alpha" version of the probe, and all data presented in this paper are from this version. A forthcoming "beta" version is planned with broadly similar specifications, but notably with an improved ADC design expected to reduce noise levels. Both the probe electronics (66) and the data acquisition system (67) have been described previously, but aspects of those reports are summarized here for clarity.

The shank is $10 \mathrm{~mm}$ long with two columns of sites with $32 \mu \mathrm{m}$ center-to-center spacing between the two columns, and $15 \mu \mathrm{m}$ center-to-center spacing along the length of the shank, for 1280 total sites on one shank (Fig 1a), or 5120 sites on the four shank probe version. The shank has a $70 \times 24 \mu \mathrm{m}$ cross-sectional profile. A stress compensation process ensures a deflection of $<100 \mu \mathrm{m}$ from base to tip on each shank. The porous TiN recording sites are $12 \times 12 \mu \mathrm{m}^{2}$ and have an impedance of $148 \pm 8 \mathrm{k} \Omega$ at $1 \mathrm{kHz}$. The tapered tip of the probe is $175 \mu \mathrm{m}$ long (tip angle $\sim 20^{\circ}$ ). Due to the planar nature of CMOS manufacturing technologies, the tip is only tapered in the two-dimensional plane of the probe; the tip shape that enters the brain is therefore a line as long as the probe thickness $(24 \mu \mathrm{m})$. However, the tip can be sharpened to form a point, using a micropipette grinder or similar at an angle as small as $15^{\circ}$ (detailed procedure not described here). The triangular tip area is covered by a single large electrode site that can be configured as an internal reference. In addition to the internal reference electrode at the tip, four of the $12 \times 12 \mu \mathrm{m}^{2}$ sites along the shank are also reserved for optional use as internal references, but their use is not recommended because of the much larger impedance of these small sites does not allow for correct cancelation of common-mode noise in all the channels. The center-to-center spacing between shanks on the four-shank version is $250 \mu \mathrm{m}$.

The probe base is mounted onto a rigid PCB that has an arrow shape (Fig 1a) with a total width of $3.5 \mathrm{~mm}$ at the thin part of the arrow near the shanks, a width of $6.9 \mathrm{~mm}$ at the thicker portion, and thickness of $\sim 1.2 \mathrm{~mm}$. The length of the rigid PCB, from shank to flexible ribbon, is $\sim 14 \mathrm{~mm}$. The base, affixed to the rigid PCB with wire bonding and epoxy, is $2.2 \times 8.7 \mathrm{~mm}^{2}$, consumes $36.5 \mathrm{~mW}$ of power, and records 384 full band $(0.5 \mathrm{~Hz}-10 \mathrm{kHz})$ signals sampled at $30 \mathrm{kHz}$ and at 14 bit resolution. The total data rate is $161.3 \mathrm{Mb} / \mathrm{s}$ (or $\sim 23.0 \mathrm{MB} / \mathrm{s}$ on disk). The mean input-referred noise level in the action potential range $(300 \mathrm{~Hz}-10 \mathrm{kHz})$ is $8.2 \mu \mathrm{V}$ root mean square, including the electrode noise (the number given in the main text, $7.2 \mu \mathrm{V}$ r.m.s., reflects the noise of the recording channel alone, which is the only aspect in which the noise of NP 1.0 and NP 2.0 differ). The input range is 12.5 $\mathrm{mV}$ peak-to-peak and the mean gain is $\sim 84$. Because of the good channel-to-channel gain uniformity (66), gain values are not calibrated for each channel separately, but per probe to reduce global process variation, and this 
calibration is applied during acquisition. Cross-talk is $0.35 \%$ on average between sites at $1 \mathrm{kHz}$ on the singleshank version and $1.51 \%$ on the four-shank version. The shank heats $<1^{\circ} \mathrm{C}$ in the brain.

The flex cable is $43.5 \mathrm{~mm}$ long, $4.0 \mathrm{~mm}$ wide, and $80 \mu \mathrm{m}$ thick. Solder pads for attaching referencing and ground connections are provided both near the top of the rigid PCB (Fig 1a, gold squares with holes on the right side of the rigid $\mathrm{PCB}$ ) as well as along two flexible "wings" on either side of the flex cable; these wings can be cut off if not used. The probe in total (shanks, base, rigid PCB, and flex cable) weighs $0.19 \mathrm{~g}$.

The headstage connects to the flex cable via a 27-pin zero insertion force (ZIF) connector. The headstage has two such connectors, one on either side of the PCB, so that two probes can be connected to a single headstage and stream data from each of their 384 channels simultaneously, for a total of 768 channels. The headstage is $10 \times 14.3 \mathrm{~mm}^{2}$ in size and weighs $0.72 \mathrm{~g}$. The headstage features a solder pad for ground, and a separate solder pad that connects directly to the tip site(s) and can be used to deliver current. The headstage has a 4-pin Omnetics connector to connect to the cable.

The cable, PXI base-station card, and software are identical to that used for NP 1.0 (67), but described briefly here for completeness. The cable has two twisted strands (each with $0.41 \mathrm{~mm}$ diameter), is $5 \mathrm{~m}$ long, weighs 5 $\mathrm{g}$, and terminates in a USB-C connector. Data from all 768 simultaneously recorded channels at $30 \mathrm{kHz}$ are transmitted across this twisted pair cable. The base-station card has four USB-C connectors, accepting input from four headstages (up to 8 probes) simultaneously, and at least two base-stations can be used together in a PXI chassis to stream data to a single computer. A single set of firmware on the base-station allows for recording interchangeably (and simultaneously) from NP 1.0 and 2.0 probes. The base station also accepts an optional digital TTL input channel for synchronization or triggering and an optional battery power supply for isolation. The probe can be configured, and data can be visualized and streamed to disk, with either SpikeGLX (https://billkarsh.github.io/SpikeGLX/) or OpenEphys (https://open-ephys.org/gui) open-source software packages.

As there are 1280 or 5120 electrode sites (i.e. physical TiN electrodes located on the shank) on the single- or 4shank probe versions respectively, but only 384 recording channels (i.e. signal processing pathways including amplification, filtering, digitization, and data transmission) available, analog switches are used to control which subset of sites is recorded at any given time. The switches are set in software and, after setting, induce a transient voltage deflection lasting $<1 \mathrm{~s}$. The switching schemes that govern which sites connect to which channels differ for the single- and four-shank probe versions, as follows.

The logic of the single-shank scheme is that each block of 32 sites maps onto consecutive groups of 32 channels, allowing for the selection of any contiguous stretch of 384 sites, given that the selected sites start with a site that is a multiple of 32 . However, within groups of 32 sites, the mapping from site to channel is scrambled such that any physically clustered group of sites maps onto a set of channels that do not correspond to any other physically clustered group of sites (Fig 5a, b). In this way, a given recorded neuron on some set of channels can be localized to one of the groups of sites that connect to those channels according to which of those groups of sites are physically clustered (see also analysis methods section "Combined bank recordings" below and Fig 5).

The logic of the four-shank scheme is that the following selections are possible: any continuous set of 384 channels on any shank; any set that includes 96 continuous sites on each of the four shanks, where the 96 sites are located at the same depth along the probe; any set that includes 96 continuous sites on each of the four shanks, but where the sites on each shank are offset by 96 from shank to shank, forming a diagonal stripe across 
the four shanks. Other selections consistent with the wiring constraints are also possible (66).

\section{Figure Captions}

Figure 1. Neuropixels 2.0 probes are miniaturized and provide high-quality recordings across thousands of sites in vivo. (A) Comparison of the NP 1.0 (top) and 2.0 (bottom) devices. NP 2.0 have four shanks (or a single shank, not shown), miniaturized rigid base and headstage, and increased recording site density (right). They allow for two probes to be attached to a single headstage (inset). (B) Example raw data traces show local field potentials and spiking signals recorded from 9 nearby recording sites in the olfactory bulb in an awake, head-fixed mouse. (C) Example spike waveforms from six selected neurons recorded on overlapping channels. The mean waveform (color) is overlaid on 50 randomly selected individual waveforms (grey). (D) Auto- and cross-correlograms (colored and black plots, respectively) of the example neurons from panel $\mathrm{C}$, shown over a -50 to +50 ms window. (E) Example spiking rasters from two NP 2.0 probes chronically implanted in a single mouse, showing spikes recorded on 6,144 sites, out of the 10,240 sites available across the two probes. Each colored block represents spike times recorded from a 'bank' of 384 channels and plotted at the depth along the probe at which they occurred. Each probe could record one bank at a time, so that two banks (768 sites), were recorded simultaneously. The 6,144 sites were accessed by altering switches in software, and recording over 8 sequential recording epochs of 768 sites. (F) Dense local recordings from dorsal striatum in head-fixed mice performing a joystick-pulling task reveal reliable sequences of spiking activity on individual trials. Left, the 384 simultaneously recorded sites (orange) cover a plane $720 \times 750 \mu \mathrm{m}$ in extent, covering a significant proportion of dorsal striatum (purple). Recording location is illustrative, and does not represent a reconstruction from histology. Right, spiking raster from ten trials reveals characteristic spiking sequences across neurons. The neurons were sorted for latency of average peak response and are shown in the same order on each trial.

Figure 2. Chronic recordings with Neuropixels $\mathbf{2 . 0}$ probes maintained high yield for $>\mathbf{8}$ weeks. (A) Stable distribution of spike amplitudes recorded across weeks (averages of $n=14$ subjects). Spike amplitude distributions from recordings made on each week are superimposed and color-coded by weeks since implantation. (B) Firing rates across channels are stable over nearly a year, in cortex (Ctx), hippocampus (HC), and thalamus $(\mathrm{TH})$ in an example recording. Spikes are spatially binned across $15 \mu \mathrm{m}$. The spike counts at each depth are normalized by the total spike count within a recording day, so that the color scale reflects the proportion of spikes found at each depth on a given day. (C) Total firing rates over the course of 60 days for all probes used in this study. A linear regression line (in $\log _{10}$ units) was fitted to the total firing rate of each probe versus days since implantation. The color of each series represents data collected in different laboratories. Note that different brain regions were targeted by each lab and each implant, likely accounting for much of the variability across implants. Nevertheless, total firing rates are unchanging or changing slowly for all tested recording targets. (D) Rate of change in log total firing rate extracted from the linear fits (slope) of each experiment in C. Each point represents one experiment. A rate of $-0.01 \mathrm{log}$ units per day indicates that over 100 days, the value declines by one log unit, i.e. a factor of 10. Filled dots represent significant correlations of the firing rate (or cluster count) with time. (E) Rate of change in log yield of spike-sorted neurons for each probe over the course of 60 days. (F) Same as $D$ for neuron yields. 
Figure 3. Post-hoc computational motion correction yields stable recordings even in the face of electrode motion. (A) Typical brain movements are parallel to the probe shank (blue arrows); they were simulated by moving the probe up and down along the same axis while recording (red arrows). (B) Movements of the brain relative to a stationary probe. A spiking raster with spikes plotted at the position they occurred along the probe. Darker spikes have larger amplitude. The shared movement of the traces across depth reveals relative motion of neurons across the whole probe over both fast ( $<1 \mathrm{~min})$ and slow ( $10 \mathrm{~min})$ timescales. (C) The motion correction algorithm counts spikes by depth and amplitude in $2 \mathrm{~s}$ time bins to create 'images' of neural activity that are registered across time. (D) The estimated position over time (colored traces) for an example recording made with imposed probe motion (black). Each color represents the position estimated at a different depth along the probe (see Fig. S5). (E) Raw data segments showing the motion correction approach. Left: a raw data segment from 14 channels where position was estimated near zero relative to the recording's zero point. Middle: raw data from a later time point where position was estimated to be $53.0 \mu \mathrm{m}$ from the zero point (a shift of $\sim 3.5$ sites). Right: the results of correcting those raw data (through resampling and spatial interpolation) to shift it to position $=0$. All spikes are shifted downward by this process, and the large spike to the left now aligns with the large spike from the first sample, presumably from the same neuron. (F) Spiking raster of a segment of an example recording with spikes plotted at the depth they occurred on the probe. The triangle-wave pattern of imposed probe motion (red) is reflected in the movement of spikes along the probe during the middle of the recording. Blue dashed box: the segment of data enlarged in panel $B$, to illustrate naturally occurring brain motion. (G) Raster of spikes detected after applying the motion correction algorithm, showing correction of both imposed motion and naturally occurring motion. (H) The motion correction algorithm improved stability measured as the absolute value of the correlation coefficient between firing rates and probe-brain motion. (I) The motion correction algorithm improved yield of neurons whose firing rates had no correlation with the imposed motion ("stable") and reduced the number whose firing rates correlated with the motion ("unstable").

Figure 4. Neuropixels 2.0 probes with motion correction allow successful recording of hundreds of neurons across days and weeks. (A) Data from an example mouse with a chronic NP 2.0 probe in visual cortex, showing significant drift between recordings on consecutive days. Plotting conventions as in Fig $3 \mathrm{~b}$. (B) Firing rate of an example neuron in response to three images presented for $1 \mathrm{~s}$ (gray box), averaged over $\mathrm{n}=5$ repetitions, on each of three days (red, green, blue) (C) Top: Average spike count response (z-scored) of the same neuron to all the images in the battery (arrows indicate the 3 example images from $b$. The responses have a correlation of 0.75 across the two days. Bottom: Example average spike count response of all units with visual fingerprint on one of the shanks on two consecutive recording days, with both neurons and images in sorted order according to similarity of responses. Color bar: z-scored response. (D) To gauge unit stability, each unit's visual fingerprint on the first day was matched with its own fingerprint and with the fingerprint of the physically closest other unit (not necessarily labeled with a consecutive index) on the second day. All units matched to themselves (points on the diagonal, 216/217), except for one that matched better with the visual fingerprint of its neighbor (single red point off the diagonal in shank 1). Gray squares separate the four shanks (numbered). (E) Same format as D, for two recordings made three weeks apart (79/88 units are matches). (F) Summary of stability of well-isolated units across 26 spliced pairs of recordings in three mice. Each point represents a single shank, and data from each mouse is shown by a different symbol. In total, 1,748 well-isolated units with visual 
fingerprints were analyzed. The estimated percentage of stable units is calculated as $2 \operatorname{Pr}(\operatorname{match})-1$ where $\operatorname{Pr}($ match) is the probability that a unit's visual fingerprint matched more closely than the nearest neighbor on the two days (see Methods for derivation). For presentation purposes only, points were jittered along the $x$-axis to avoid overlaps. Note that the interval between implantation and the first recording in each pair of recordings was variable, in some cases exceeding 6 months.

Figure 5. Neuropixels 2.0 probes allow recording from twice as many sites as the number of recording channels. (A) Sites from multiple banks connect to a single set of recording channels, showing 10 sites out of 384 for each bank. Software controls allow switching the channels to the sites in bank 1 (left), in bank 2 (middle) or in both banks concurrently (right). To allow unmixing, the mapping from bank 2 sites to channels is scrambled relative to bank 1. (B) Spiking raster (conventions as in Fig 3b) from recordings with all three configurations. When bank 1 and bank 2 are recorded together (right), spikes are plotted at their inferred locations based on the mismatch score of their source template. As expected, in this condition the spike amplitudes are lower by a factor of two.

\section{Acknowledgements}

Funding: This research program was funded by the Wellcome Trust (204915/Z/16/Z), the Howard Hughes Medical Institute Janelia Research Campus, the European Research Council (ERC Synergy Grant 951319 to E.I.M.), the Research Council of Norway (to E.I.M.: NORBRAIN grant no. 295721; FRIPRO grant no. 286225; Centre of Excellence grant no. 223262), the Kavli Foundation (E.I.M.), the Neuroelectronics Research Flanders, and the Champalimaud Foundation.

Additional support was provided by the Klingenstein-Simons Foundation (N.A.S.), the Pew Biomedical Scholars program (N.A.S.), the National Institutes of Health (1U01NS113252-01 to N.A.S.), the Research Foundation Flanders (FWO, G096219N, to S.H.), KU Leuven (C14/17/109 to S.H.), the BBSRC (BB/P020607/1 to M.O.), the Academy of Medical Sciences and Wellcome Trust (Springboard award SBF002\1045 to M.O.), the Sainsbury Wellcome Centre for Neural Circuits and Behaviour (A.L.), and the Hermesfonds with a VLAIO Baekeland mandate (HBC.2018.2114 to R.v.D.). M.C. holds the GlaxoSmithKline / Fight for Sight Chair in Visual Neuroscience.

\section{Author contributions:}




\begin{tabular}{|c|c|c|c|c|c|c|c|c|c|c|c|c|c|c|c|c|c|c|c|c|c|c|c|c|c|c|c|c|c|c|c|c|c|c|c|c|c|c|}
\hline Conceptualization & $\bullet$ & & & & & & & & & & & & & & & & & & & & & & & & & & $\bullet$ & & $\bullet$ & & & $\bullet$ & & & $\bullet$ & $\bullet$ & $\bullet$ & $\bullet$ \\
\hline Data preprocessing & $\bullet$ & • & $\bullet$ & - & - & $\bullet$ & • & $\bullet$ & $\bullet$ & & & • & $\bullet$ & & $\bullet$ & & & $\bullet$ & & $\bullet$ & & & & & $\bullet$ & & & & & & & & & & & & & \\
\hline Data analysis & $\bullet$ & • & $\bullet$ & - & - & & • & $\bullet$ & & & & $\bullet$ & & & $\bullet$ & & & • & & & & & & & & $\bullet$ & & & $\bullet$ & & & & & & & & & \\
\hline Data collection: chronic & & - & $\bullet$ & $\cdot$ & & $\bullet$ & & & • & $\bullet$ & & & $\bullet$ & & & & & & & • & & $\bullet$ & & & & & & & & & & & & & & & & \\
\hline Data collection: acute & $\bullet$ & & & & & & $\bullet$ & & & & $\bullet$ & & & & & & & $\bullet$ & & & & & & & $\bullet$ & & & & & & & & & & & & & \\
\hline Software development & & & & & $\bullet$ & & & & & & & $\bullet$ & & $\bullet$ & & & & & & & & & & & & & & & & & & & & & & & & \\
\hline Software beta testing & $\bullet$ & & & & & & & $\bullet$ & & & $\bullet$ & & $\bullet$ & & & & & & & & & & & & & & & & & & & & & & & & & \\
\hline Electronics hardware design & & & & & & & & & & & & & & & & $\bullet$ & $\bullet$ & & $\bullet$ & & & & $\bullet$ & $\bullet$ & & & $\bullet$ & & & & & & & & & & & \\
\hline Implant hardware design & & $\bullet$ & $\bullet$ & $\bullet$ & & & . & & & & & & & & $\bullet$ & & & & & & $\bullet$ & & & & & & & & & & & & & & & $\bullet$ & & \\
\hline Visualization & $\cdot$ & - & $\bullet$ & $\bullet$ & $\bullet$ & & • & & & & & & & & $\bullet$ & & & & & & & & & & & $\bullet$ & & & & & & & & & & & $\bullet$ & \\
\hline Writing: original draft & $\bullet$ & • & $\bullet$ & $\bullet$ & - & & . & & & & & & & & & & & & & & & & & & & & & & & & & & & & & & $\bullet$ & \\
\hline Writing: methods & $\bullet$ & $\bullet$ & $\bullet$ & $\bullet$ & $\bullet$ & $\bullet$ & $\bullet$ & & $\bullet$ & & $\bullet$ & $\bullet$ & $\bullet$ & & & & & $\bullet$ & & $\bullet$ & & & & & & & & & & & & & & & & & & \\
\hline Writing: review and editing & $\bullet$ & $\bullet$ & $\cdot$ & $\cdot$ & $\bullet$ & $\bullet$ & $\bullet$ & & & & $\bullet$ & $\cdot$ & $\bullet$ & $\cdot$ & $\cdot$ & $\bullet$ & & & $\bullet$ & $\bullet$ & $\bullet$ & $\bullet$ & & $\bullet$ & $\bullet$ & $\bullet$ & $\bullet$ & & $\bullet$ & $\bullet$ & $\bullet$ & & & & & & $\bullet$ & - \\
\hline Supervision & $\bullet$ & & & $\bullet$ & & & & & & & & & & & $\bullet$ & $\bullet$ & & & & & & & & & & $\bullet$ & $\bullet$ & $\bullet$ & $\bullet$ & $\bullet$ & $\bullet$ & $\bullet$ & $\bullet$ & $\bullet$ & $\bullet$ & $\bullet$ & $\bullet$ & $\bullet$ \\
\hline Funding acquisition & & & & & & & & & & & & & & & & & & & & & & & & & & & & & & & $\bullet$ & • & $\bullet$ & & • & $\bullet$ & $\bullet$ & - \\
\hline
\end{tabular}

Competing interests: Authors B. Dutta, C. Mora-Lopez, J. O'Callaghan, J. Putzeys, S. Wang, and M. Welkenhuysen are employees of IMEC, which sells Neuropixels probes.

Data and materials availability: The datasets with imposed motion (Fig 3) are shared at Figshare (data upload is ongoing - link in preparation). It is not practical to share the raw data from chronic recordings, but other datasets besides those shared above will be made available by request where possible.

\section{Supplementary material includes:}

Materials and Methods

Figs. S1 to S5

Tables S1

References (68-76)

\section{References}

1. R. Chen, A. Canales, P. Anikeeva, Neural recording and modulation technologies. Nat. Rev. Mater. 2, 116 (2017).

2. J. P. Seymour, F. Wu, K. D. Wise, E. Yoon, State-of-the-art MEMS and microsystem tools for brain research. Microsyst. Nanoeng. 3, 1-16 (2017).

3. N. A. Steinmetz, C. Koch, K. D. Harris, M. Carandini, Challenges and opportunities for large-scale electrophysiology with Neuropixels probes. Curr. Opin. Neurobiol. 50, 92-100 (2018).

4. G. Hong, C. M. Lieber, Novel electrode technologies for neural recordings. Nat. Rev. Neurosci. 20, 330345 (2019).

5. D. Kleinfeld, L. Luan, P. P. Mitra, J. T. Robinson, R. Sarpeshkar, K. Shepard, C. Xie, T. D. Harris, Can One Concurrently Record Electrical Spikes from Every Neuron in a Mammalian Brain? Neuron. 103, 1005-1015 (2019).

6. J. J. Jun, N. A. Steinmetz, J. H. Siegle, D. J. Denman, M. Bauza, B. Barbarits, A. K. Lee, C. A. 
Anastassiou, A. Andrei, Ç. Aydın, M. Barbic, T. J. Blanche, V. Bonin, J. Couto, B. Dutta, S. L. Gratiy, D. A. Gutnisky, M. Häusser, B. Karsh, P. Ledochowitsch, C. M. Lopez, C. Mitelut, S. Musa, M. Okun, M. Pachitariu, J. Putzeys, P. D. Rich, C. Rossant, W.-L. Sun, K. Svoboda, M. Carandini, K. D. Harris, C. Koch, J. O'Keefe, T. D. Harris, Fully integrated silicon probes for high-density recording of neural activity. Nature. 551, 232-236 (2017).

7. B. C. Raducanu, R. F. Yazicioglu, C. M. Lopez, M. Ballini, J. Putzeys, S. Wang, A. Andrei, V. Rochus, M. Welkenhuysen, N. van Helleputte, Time multiplexed active neural probe with 1356 parallel recording sites. Sensors. 17, 2388 (2017).

8. W. E. Allen, M. Z. Chen, N. Pichamoorthy, R. H. Tien, M. Pachitariu, L. Luo, K. Deisseroth, Thirst regulates motivated behavior through modulation of brainwide neural population dynamics. Science. 364 (2019), doi:10.1126/science.aav3932.

9. J. H. Siegle, X. Jia, S. Durand, S. Gale, C. Bennett, N. Graddis, G. Heller, T. K. Ramirez, H. Choi, J. A. Luviano, A survey of spiking activity reveals a functional hierarchy of mouse corticothalamic visual areas. Biorxiv, 805010 (2019).

10. N. A. Steinmetz, P. Zatka-Haas, M. Carandini, K. D. Harris, Distributed coding of choice, action and engagement across the mouse brain. Nature, 1-8 (2019).

11. C. Stringer, M. Pachitariu, N. Steinmetz, C. B. Reddy, M. Carandini, K. D. Harris, Spontaneous behaviors drive multidimensional, brainwide activity. Science. 364, 255-255 (2019).

12. D. A. Evans, A. V. Stempel, R. Vale, S. Ruehle, Y. Lefler, T. Branco, A synaptic threshold mechanism for computing escape decisions. Nature. 558, 590-594 (2018).

13. M. Vélez-Fort, E. F. Bracey, S. Keshavarzi, C. V. Rousseau, L. Cossell, S. C. Lenzi, M. Strom, T. W. Margrie, A circuit for integration of head-and visual-motion signals in layer 6 of mouse primary visual cortex. Neuron. 98, 179-191 (2018).

14. C. Bennett, S. D. Gale, M. E. Garrett, M. L. Newton, E. M. Callaway, G. J. Murphy, S. R. Olsen, Higherorder thalamic circuits channel parallel streams of visual information in mice. Neuron. 102, 477-492 (2019).

15. D. Kostadinov, M. Beau, M. Blanco-Pozo, M. Häusser, Predictive and reactive reward signals conveyed by climbing fiber inputs to cerebellar Purkinje cells. Nat. Neurosci. 22, 950-962 (2019).

16. S. Musall, M. T. Kaufman, A. L. Juavinett, S. Gluf, A. K. Churchland, Single-trial neural dynamics are dominated by richly varied movements. Nat. Neurosci. 22, 1677-1686 (2019).

17. J. Park, J. W. Phillips, K. A. Martin, A. W. Hantman, J. T. Dudman, Flexible routing of motor control signals through neocortical projection neuron classes. bioRxiv, 772517 (2019).

18. S. Schröder, N. A. Steinmetz, M. Krumin, M. Pachitariu, M. Rizzi, L. Lagnado, K. D. Harris, M. Carandini, Retinal outputs depend on behavioural state. BioRxiv, 638049 (2019).

19. C. Stringer, M. Pachitariu, N. Steinmetz, M. Carandini, K. D. Harris, High-dimensional geometry of population responses in visual cortex. Nature, 1 (2019).

20. L. D. Liu, S. Chen, M. N. Economo, N. Li, K. Svoboda, Accurate localization of linear probe electrodes across multiple brains. bioRxiv (2020).

21. B. A. Sauerbrei, J.-Z. Guo, J. D. Cohen, M. Mischiati, W. Guo, M. Kabra, N. Verma, B. Mensh, K. Branson, A. W. Hantman, Cortical pattern generation during dexterous movement is input-driven. Nature. 577, 386-391 (2020).

22. J. Krupic, M. Bauza, S. Burton, J. O'Keefe, Local transformations of the hippocampal cognitive map. Science. 359, 1143-1146 (2018).

23. R. J. Gardner, L. Lu, T. Wernle, M.-B. Moser, E. I. Moser, Correlation structure of grid cells is preserved during sleep. Nat. Neurosci. 22, 598-608 (2019).

24. C. Böhm, A. K. Lee, bioRxiv, in press, doi:10.1101/2020.09.11.292888.

25. T. Z. Luo, A. G. Bondy, D. Gupta, V. A. Elliott, C. D. Kopec, C. D. Brody, An approach for long-term, multiprobe Neuropixels recordings in unrestrained rats. eLife. 9, e59716 (2020).

26. Q. Gaucher, M. Panniello, A. Z. Ivanov, J. C. Dahmen, A. J. King, K. M. M. Walker, Complexity of frequency receptive fields predicts tonotopic variability across species. eLife. 9, e53462 (2020).

27. E. M. Trautmann, S. D. Stavisky, S. Lahiri, K. C. Ames, M. T. Kaufman, D. J. O'Shea, S. Vyas, X. Sun, S. 
I. Ryu, S. Ganguli, K. V. Shenoy, Accurate Estimation of Neural Population Dynamics without Spike Sorting. Neuron. 103, 292-308.e4 (2019).

28. T.-M. Fu, G. Hong, R. D. Viveros, T. Zhou, C. M. Lieber, Highly scalable multichannel mesh electronics for stable chronic brain electrophysiology. Proc. Natl. Acad. Sci. 114, E10046-E10055 (2017).

29. J. E. Chung, H. R. Joo, J. L. Fan, D. F. Liu, A. H. Barnett, S. Chen, C. Geaghan-Breiner, M. P. Karlsson, M. Karlsson, K. Y. Lee, H. Liang, J. F. Magland, J. A. Pebbles, A. C. Tooker, L. F. Greengard, V. M. Tolosa, L. M. Frank, High-Density, Long-Lasting, and Multi-region Electrophysiological Recordings Using Polymer Electrode Arrays. Neuron. 101, 21-31.e5 (2019).

30. E. Musk, An integrated brain-machine interface platform with thousands of channels. J. Med. Internet Res. 21, e16194 (2019).

31. G. Guitchounts, J. E. Markowitz, W. A. Liberti, T. J. Gardner, A carbon-fiber electrode array for long-term neural recording. J. Neural Eng. 10, 046016 (2013).

32. L. Luan, X. Wei, Z. Zhao, J. J. Siegel, O. Potnis, C. A. Tuppen, S. Lin, S. Kazmi, R. A. Fowler, S. Holloway, A. K. Dunn, R. A. Chitwood, C. Xie, Ultraflexible nanoelectronic probes form reliable, glial scarfree neural integration. Sci. Adv. 3, e1601966 (2017).

33. D. Egert, J. R. Pettibone, S. Lemke, P. R. Patel, C. M. Caldwell, D. Cai, K. Ganguly, C. A. Chestek, J. Berke, bioRxiv, in press, doi:10.1101/2020.05.26.117572.

34. E. J. Welle, P. R. Patel, J. E. Woods, A. Petrossians, E. della Valle, A. Vega-Medina, J. M. Richie, D. Cai, J. D. Weiland, C. A. Chestek, Ultra-small carbon fiber electrode recording site optimization and improved in vivo chronic recording yield. J. Neural Eng. 17, 026037 (2020).

35. M. Recce, J. O'Keefe, in Soc Neuroci Abstr (1989), vol. 15, p. 1250.

36. A. K. Dhawale, R. Poddar, S. B. Wolff, V. A. Normand, E. Kopelowitz, B. P. Ölveczky, Automated longterm recording and analysis of neural activity in behaving animals. Elife. 6, e27702 (2017).

37. E. M. Maynard, C. T. Nordhausen, R. A. Normann, The Utah Intracortical Electrode Array: A recording structure for potential brain-computer interfaces. Electroencephalogr. Clin. Neurophysiol. 102, 228-239 (1997).

38. C. A. Chestek, V. Gilja, P. Nuyujukian, J. D. Foster, J. M. Fan, M. T. Kaufman, M. M. Churchland, Z. Rivera-Alvidrez, J. P. Cunningham, S. I. Ryu, K. V. Shenoy, Long-term stability of neural prosthetic control signals from silicon cortical arrays in rhesus macaque motor cortex. J. Neural Eng. 8, 045005 (2011).

39. M. Okun, A. Lak, M. Carandini, K. D. Harris, Long term recordings with immobile silicon probes in the mouse cortex. PLoS One. 11, e0151180 (2016).

40. J.-O. Muthmann, A. J. Levi, H. C. Carney, A. C. Huk, bioRxiv, in press, doi:10.1101/2020.08.09.243279.

41. C. E. Schoonover, S. N. Ohashi, R. Axel, A. J. P. Fink, bioRxiv, in press, doi:10.1101/2020.09.24.312132.

42. A. L. Juavinett, G. Bekheet, A. K. Churchland, Chronically implanted Neuropixels probes enable highyield recordings in freely moving mice. eLife. 8, e47188 (2019).

43. A. A. Emondi, S. P. Rebrik, A. V. Kurgansky, K. D. Miller, Tracking neurons recorded from tetrodes across time. J. Neurosci. Methods. 135, 95-105 (2004).

44. A. Jackson, E. E. Fetz, Compact Movable Microwire Array for Long-Term Chronic Unit Recording in Cerebral Cortex of Primates. J. Neurophysiol. 98, 3109-3118 (2007).

45. A. S. Tolias, A. S. Ecker, A. G. Siapas, A. Hoenselaar, G. A. Keliris, N. K. Logothetis, Recording Chronically From the Same Neurons in Awake, Behaving Primates. J. Neurophysiol. 98, 3780-3790 (2007).

46. A. S. Dickey, A. Suminski, Y. Amit, N. G. Hatsopoulos, Single-Unit Stability Using Chronically Implanted Multielectrode Arrays. J. Neurophysiol. 102, 1331-1339 (2009).

47. G. W. Fraser, A. B. Schwartz, Recording from the same neurons chronically in motor cortex. J. Neurophysiol. 107, 1970-1978 (2011).

48. D. B. T. McMahon, I. V. Bondar, O. A. T. Afuwape, D. C. Ide, D. A. Leopold, One month in the life of a neuron: longitudinal single-unit electrophysiology in the monkey visual system. APSselect. 1, 1748-1762 (2014).

49. J. L. Shobe, L. D. Claar, S. Parhami, K. I. Bakhurin, S. C. Masmanidis, Brain activity mapping at multiple 
scales with silicon microprobes containing 1,024 electrodes. J. Neurophysiol. 114, 2043-2052 (2015).

50. G. Rios, E. V. Lubenov, D. Chi, M. L. Roukes, A. G. Siapas, Nanofabricated Neural Probes for Dense 3-D Recordings of Brain Activity. Nano Lett. 16, 6857-6862 (2016).

51. J. Scholvin, J. P. Kinney, J. G. Bernstein, C. Moore-Kochlacs, N. Kopell, C. G. Fonstad, E. S. Boyden, Close-Packed Silicon Microelectrodes for Scalable Spatially Oversampled Neural Recording. IEEE Trans. Biomed. Eng. 63, 120-130 (2016).

52. M. S. Saleh, S. M. Ritchie, M. A. Nicholas, R. Bezbaruah, J. W. Reddy, M. Chamanzar, E. A. Yttri, R. P. Panat, CMU Array: A 3D Nano-Printed, Customizable Ultra-High-Density Microelectrode Array Platform. bioRxiv, 742346 (2019).

53. A. Obaid, M.-E. Hanna, Y.-W. Wu, M. Kollo, R. Racz, M. R. Angle, J. Müller, N. Brackbill, W. Wray, F. Franke, E. J. Chichilnisky, A. Hierlemann, J. B. Ding, A. T. Schaefer, N. A. Melosh, Massively parallel microwire arrays integrated with CMOS chips for neural recording. Sci. Adv. 6, eaay2789 (2020).

54. K. Sahasrabuddhe, A. A. Khan, A. P. Singh, T. M. Stern, Y. Ng, A. Tadić, P. Orel, C. LaReau, D. Pouzzner, K. Nishimura, K. M. Boergens, S. Shivakumar, M. S. Hopper, B. Kerr, M.-E. S. Hanna, R. J. Edgington, I. McNamara, D. Fell, P. Gao, A. Babaie-Fishani, S. Veijalainen, A. V. Klekachev, A. M. Stuckey, B. Luyssaert, T. D. Y. Kozai, C. Xie, V. Gilja, B. Dierickx, Y. Kong, M. Straka, H. S. Sohal, M. R. Angle, "The Argo: A 65,536 channel recording system for high density neural recording in vivo" (preprint, Neuroscience, 2020), , doi:10.1101/2020.07.17.209403.

55. K. Ohki, S. Chung, Y. H. Ch'ng, P. Kara, R. C. Reid, Functional imaging with cellular resolution reveals precise micro-architecture in visual cortex. Nature. 433, 597-603 (2005).

56. S.-C. Yen, J. Baker, C. M. Gray, Heterogeneity in the responses of adjacent neurons to natural stimuli in cat striate cortex. J. Neurophysiol. 97, 1326-1341 (2007).

57. V. Bonin, M. H. Histed, S. Yurgenson, R. C. Reid, Local diversity and fine-scale organization of receptive fields in mouse visual cortex. J. Neurosci. 31, 18506-18521 (2011).

58. B. M. Kampa, M. M. Roth, W. Göbel, F. Helmchen, Representation of visual scenes by local neuronal populations in layer 2/3 of mouse visual cortex. Front. Neural Circuits. 5, 18 (2011).

59. K. A. Martin, S. Schröder, Functional heterogeneity in neighboring neurons of cat primary visual cortex in response to both artificial and natural stimuli. J. Neurosci. 33, 7325-7344 (2013).

60. I. V. Bondar, D. A. Leopold, B. J. Richmond, J. D. Victor, N. K. Logothetis, Long-term stability of visual pattern selective responses of monkey temporal lobe neurons. PloS One. 4, e8222 (2009).

61. D. Deitch, A. Rubin, Y. Ziv, bioRxiv, in press, doi:10.1101/2020.10.05.327049.

62. T. D. Marks, M. J. Goard, Stimulus-dependent representational drift in primary visual cortex. bioRxiv (2020).

63. K. H. Lee, Y.-L. Ni, M. Meister, Electrode pooling: How to boost the yield of switchable silicon probes for neuronal recordings. bioRxiv, 851691 (2019).

64. C. Gold, D. A. Henze, C. Koch, G. Buzsaki, On the origin of the extracellular action potential waveform: a modeling study. J. Neurophysiol. 95, 3113-3128 (2006).

65. R. Fiáth, B. C. Raducanu, S. Musa, A. Andrei, C. M. Lopez, M. Welkenhuysen, P. Ruther, A. Aarts, I. Ulbert, Fine-scale mapping of cortical laminar activity during sleep slow oscillations using high-density linear silicon probes. J. Neurosci. Methods. 316, 58-70 (2019).

66. S. Wang, S. K. Garakoui, H. Chun, D. G. Salinas, W. Sijbers, J. Putzeys, E. Martens, J. Craninckx, N. Van Helleputte, C. M. Lopez, A Compact Quad-Shank CMOS Neural Probe With 5,120 Addressable Recording Sites and 384 Fully Differential Parallel Channels. IEEE Trans. Biomed. Circuits Syst. 13, 1625-1634 (2019).

67. J. Putzeys, B. C. Raducanu, A. Carton, J. De Ceulaer, B. Karsh, J. H. Siegle, N. Van Helleputte, T. D. Harris, B. Dutta, S. Musa, C. Mora Lopez, Neuropixels Data-Acquisition System: A Scalable Platform for Parallel Recording of 10 000+ Electrophysiological Signals. IEEE Trans. Biomed. Circuits Syst. 13, 16351644 (2019).

68. J. E. Osborne, J. T. Dudman, RIVETS: a mechanical system for in vivo and in vitro electrophysiology and imaging. PloS One. 9, e89007 (2014).

69. B. Panigrahi, K. A. Martin, Y. Li, A. R. Graves, A. Vollmer, L. Olson, B. D. Mensh, A. Y. Karpova, J. T. 
Dudman, Dopamine is required for the neural representation and control of movement vigor. Cell. 162, 1418-1430 (2015).

70. L. Madisen, T. Mao, H. Koch, J. Zhuo, A. Berenyi, S. Fujisawa, Y.-W. A. Hsu, A. J. Garcia, X. Gu, S. Zanella, J. Kidney, H. Gu, Y. Mao, B. M. Hooks, E. S. Boyden, G. Buzsáki, J. M. Ramirez, A. R. Jones, K. Svoboda, X. Han, E. E. Turner, H. Zeng, A toolbox of Cre-dependent optogenetic transgenic mice for light-induced activation and silencing. Nat. Neurosci. 15, 793-802 (2012).

71. D. G. Krige, A statistical approach to some basic mine valuation problems on the Witwatersrand. J. South. Afr. Inst. Min. Metall. 52, 119-139 (1951).

72. J. W. Pillow, J. Shlens, E. Chichilnisky, E. P. Simoncelli, A model-based spike sorting algorithm for removing correlation artifacts in multi-neuron recordings. PloS One. 8, e62123 (2013).

73. M. Pachitariu, N. Steinmetz, S. Kadir, M. Carandini, Kilosort: realtime spike-sorting for extracellular electrophysiology with hundreds of channels. BioRxiv, 061481 (2016).

74. H. Foroosh, J. B. Zerubia, M. Berthod, Extension of phase correlation to subpixel registration. IEEE Trans. Image Process. 11, 188-200 (2002).

75. M. Pachitariu, C. Stringer, M. Dipoppa, S. Schröder, L. F. Rossi, H. Dalgleish, M. Carandini, K. D. Harris, Suite2p: beyond 10,000 neurons with standard two-photon microscopy. Biorxiv (2017).

76. C. Rossant, S. N. Kadir, D. F. Goodman, J. Schulman, M. L. Hunter, A. B. Saleem, A. Grosmark, M. Belluscio, G. H. Denfield, A. S. Ecker, Spike sorting for large, dense electrode arrays. Nat. Neurosci. 19, 634 (2016). 